Manuscript submitted to:

AIMS Genetics
Volume 2, Issue 1, 1-12.

DOI: $10.3934 /$ genet.2015.1.1

Received date 30 October 2014, Accepted date 16 December 2014, Published date 25 December 2014

\title{
Review
}

\section{Drosophila as a model for chromosomal instability}

\author{
Dawei Liu, Zeeshan Shaukat, Rashid Hussain, Mahwish Khan and Stephen L. Gregory * \\ School of Molecular and Biomedical Science, University of Adelaide, North Terrace, Adelaide S.A. \\ 5006, Australia
}

* Correspondence: Email: stephen.gregory@adelaide.edu.au; Tel: +61 8 83137536; Fax: +61883137534 .

\begin{abstract}
Chromosomal instability (CIN) is a common feature of tumours that leads to increased genetic diversity in the tumour and poor clinical outcomes. There is considerable interest in understanding how CIN comes about and how its contribution to drug resistance and metastasis might be counteracted. In the last decade a number of CIN model systems have been developed in Drosophila that offer unique benefits both in understanding the development of CIN in a live animal as well as giving the potential to do genome wide screens for therapeutic candidate genes. This review outlines the mechanisms used in several Drosophila CIN model systems and summarizes some significant outcomes and opportunities that they have produced.
\end{abstract}

Keywords: aneuploidy; cell cycle; checkpoint; chromosomal instability; DNA damage; Drosophila; JNK; metabolism; ROS

\section{Introduction}

The acquisition of mutations is a driving force in the formation of any tumour, as these mutations represent the genetic diversity from which aberrantly proliferative cells can emerge. Genomic instability, or an increased mutation rate, can be generated by numerous cellular defects, such as lack of DNA repair, and these typically give a strong predisposition to tumorigenesis [1]. Chromosomal instability, or CIN, refers to an increased rate of DNA changes on the largest scale: gain or loss of whole chromosomes or chromosome sections. As a common form of genomic instability, CIN is linked to tumorigenesis, particularly in solid tumours, where the frequency of chromosomal aberrations can be as high as $90 \%$ [2]. There are several reasons why CIN might be found so often in tumours: some common oncogenic mutations tend to promote CIN by disrupting mitosis, some tumours typically only progress with the gain or loss of specific chromosome arms, and for a tumour to go on to metastasize seems to require the kind of dramatic rearrangements that 
CIN generates [3]. Inducing CIN in otherwise normal mouse models is able to double the rate of spontaneous tumour formation [4], and in human cancers CIN is associated with significantly worse clinical outcomes [5]. Because CIN generates genetic diversity in tumours it is thought to promote drug resistance and relapses following chemotherapy. For these reasons there has been considerable research into the causes and possible therapies for chromosomal instability.

One of the problems in trying to understand the onset of CIN in tumours is that by the time they are detected, they have divided unstably many times and become so genetically diverse that it is hard to identify the specific changes that induced the CIN. In theory, by examining many clinical CIN tumour samples we should be able to find the common changes, but the limited studies available have not clearly identified causal mutations [6]. An alternative approach, testing specific mitotic defects in culture or animal models, has shown that CIN can be caused by a range of defects in the attachment of chromosomes to the spindle as well as by loss of checkpoints, elevated DNA damage or replication stress [7]. This wide range of possible mechanisms may explain why it has been difficult to identify individual causal mutations in specific tumours, and also raises clinical challenges. It may be obvious that CIN is a dangerous cellular phenotype that we would like to prevent, but CIN prevention is problematic even in theory, with so many possible defects that can cause instability. An alternative is to accept that CIN will arise in tumours, and to look instead for therapies that can target such cells. From this perspective, the CIN phenotype is a therapeutic advantage, because CIN represents a significant difference from normal proliferating cells that may allow targeting of therapy to avoid damaging the patient. The question then is: can we find targets that can be disrupted to specifically induce death in CIN cells without affecting normal dividing cells?

\section{CIN models and their limitations}

An obvious approach to this problem is to get cultures of CIN cells, and screen them with chemical libraries to find anything that kills CIN, but not normal cells. To some extent this has been attempted [8], but there are several challenges with this approach. CIN lines are inherently varied-potentially every cell has a different genome, and each cell varies as it is cultured. This means that it is problematic to do reproducible experiments. However, the objective is to find generalizable therapies that affect any CIN cells, so in theory the approach can still work if enough chemicals are tested enough times. Using karyotypic heterogeneity as a proxy for CIN, the well characterized panel of 60 human cancer cell lines from the NCI has been used to identify drugs that preferentially affect karyotypically diverse cell lines [9,10]. Perhaps unsurprisingly, none of the current cancer therapeutics tested were particularly effective against the unstable cell lines. Some novel drug classes were found that could inhibit the growth of unstable lines with some specificity, however, as the authors comment, these correlations are a "blunt tool for drug discovery requiring secondary experimental confirmation" [10]. In addition, the cell lines used have inevitably arisen with constraints very different to those faced by tumours in vivo, and the most effective chemicals identified gave no clear idea of how they might be working. For these reasons it is valuable to have animal CIN tumour models in which specific and reproducible mechanisms for killing CIN cells can be identified and characterized. This review will focus on the development of CIN models in Drosophila, discussing the relative merits of this system and the progress that has been achieved so far. 


\section{Advantages of using Drosophila}

As an animal model for studying CIN, Drosophila has some significant advantages. It is now straightforward to manipulate the expression of any gene in Drosophila, at any stage of development in any tissue of interest. In the context of CIN, this means that animals can be grown that are genetically normal, with CIN induced by gene mis-expression in the proliferating tissue of choice when required. For example, the larval wing disc can be used to provide a testbed of cells that start out identical each time the experiment is done, while allowing the generation of a diverse set of CIN cells to study $[11,12,13]$. This avoids the issues of reproducibility faced when using CIN cell lines, as well as providing an in vivo epithelium that better reflects the environment of CIN tumours when they arise. This could theoretically be done in mice $[14,15]$, but large scale screening in mice is impracticably time consuming and expensive. To identify and characterize novel mechanisms by which CIN cells can be specifically killed, it is useful to have an inducible in vivo system that allows genome-wide screening. Using Drosophila provides such a system as well as giving access to unparalleled resources for genetic analysis of any candidates identified [16]. This includes easy screening for genetic interactions, pathway dissection by epistasis and modelling of most of the hallmarks of cancer [17-21].

\section{Drosophila CIN models}

As mentioned above, there are numerous mitotic processes that are known to give CIN when disrupted in cell culture or mouse models, and several of these have been exploited in Drosophila to create inducible CIN models. They can be broadly classified as disruption of the mitotic spindle, checkpoints, cytokinesis or DNA repair.

\subsection{Mitotic spindle disruption}

Defects in spindle structure or kinetochore dynamics represent straightforward mechanisms for generating CIN. For example, if the spindle is incorrectly formed with too many poles, then chromosome segregation is affected (Figure 1A). Centrosome number is frequently aberrant in cancers, so this form of CIN has been modelled by altering the levels of centrosome regulatory genes such as polo or sak, and showing that neural cells with aberrant numbers of centrosomes go on to form malignant and metastatic tumours in Drosophila [22,23]. It is interesting that although very little CIN was initially detected in the mutant brains, when they had been transplanted and grown in a host they became strikingly aneuploid. Exactly when and how the CIN arises in these models is not well characterized, but they appear to recapitulate human tumorigenesis in which CIN is generally not an early trigger, but arises in an already hyper-proliferative tissue. Another striking observation from cells with elevated centrosome numbers was that spindles are surprisingly effective at generating a bipolar array even with many extra centrosomes present, as long as the spindle checkpoint is working to allow enough time to cluster the centrosomes [23,24]. This checkpoint control is not completely effective in neuroblasts, though, which can lose the correct spindle alignment and cell polarity, leading to a failure to differentiate and consequent overproliferation. Interestingly, CIN tumours can also be made without altering the centrosomes simply by disrupting neuroblast polarity. In this case, again there is a failure to differentiate, and an expansion of 


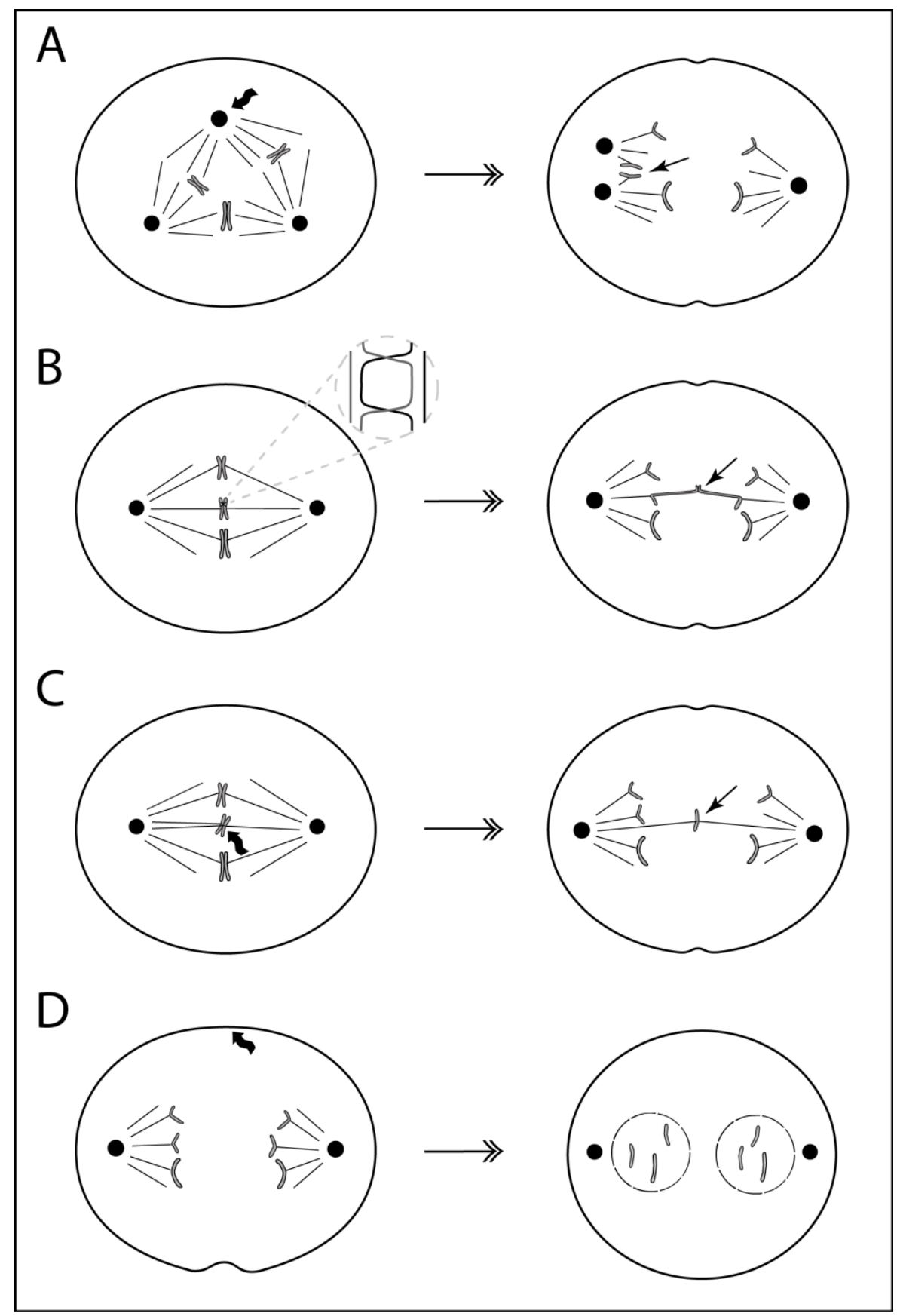

Figure 1. Drosophila CIN models: (A) An induced defect in centrosome number (wavy arrow) leads to a tripolar spindle. This is resolved into a bipolar array before anaphase, but one chromosome fails to segregate (arrow). (B) An induced defect in DNA damage repair or replication leads to still catenated chromatids (enlarged). At anaphase this leads to a chromosome bridge (arrow) that will break, leading to loss or gain of chromosome segments. (C) An induced spindle checkpoint defect means that a kinetochore attached to both poles (wavy arrow) may not be resolved before anaphase, leading to failure to segregate a lagging chromosome (arrow) that is attached to both poles. (D) An induced cytokinesis defect (wavy arrow) leads to no separation of daughter cells, and results in a binucleate cell with a tetraploid genome and an extra centrosome. 
proliferative neuroblasts that can be transplanted to form malignant and metastatic tumours [25]. The initial neuroblasts are karyotypically normal, but become aneuploid and acquire centrosome defects as the tumour develops. From these studies and others [26] it seems that most tissues in Drosophila resist tumorigenesis from centrosome alterations by dying, but neuroblasts are an exception. In these cells, anything that expands the 'stem-like' population of transit amplifying lineage neuroblasts will tend to not only cause overgrowth, but also the acquisition of additional defects that cause CIN and allow metastatic tumour growth. It will be interesting to find out what changes arise so rapidly and frequently to transform overgrowing neuroblasts; recent work in this direction has implicated DNA damage [27].

\subsection{Elevated DNA damage}

Mutations that either increase the rate of DNA damage or that reduce the cell's ability to repair DNA damage are another type of CIN model available in Drosophila (Figure 1B). For example, high levels of instability can be induced by blocking tefu, the Drosophila homolog of ATM, which is needed for DNA damage repair and telomere maintenance [28]. Interestingly, ATM also acts as a redox sensor protein, so it is activated either by DNA damage itself or by oxidative stress that threatens the DNA [29]. Balancing the levels of cellular pro- and anti-oxidants is clearly important for cells, as too much of either is able to generate CIN [30]. Furthermore, the aneuploidy generated by CIN is known to cause oxidative stress [31], which can damage DNA to cause further aneuploidy, so there is potential for feedback reinforcement of initially minor oxidative insults. Sophisticated tools are now available in Drosophila to monitor the levels and locations of redox stress in live tissues [32]. DNA damage itself is harder to monitor live, but some markers are available [33,34]. It would be particularly useful to be able to monitor the persistence of DNA damage into mitosis, as there are many unresolved questions about chromosome bridges and the resolution of double strand breaks in mitosis. For example, replication stress can induce CIN [35], but it is not clear whether this mechanism is often found in CIN tumours [36]. Ultrafine anaphase bridges, which result from late decatenation of chromatids [37], represent another potential source of CIN that has not yet been explored in Drosophila. These bridges indicate an unexpected level of DNA processing during mitosis even after the DNA damage and spindle checkpoints have been passed. This may explain why mitotic stability is so dependent on DNA damage repair being completed in time. The need for efficient DNA repair has been highlighted by our work showing that CIN cells are particularly dependent on JNK signalling in G2 to prevent DNA damage accumulation and apoptosis [38]. Even just altering G2 duration strongly affects the survival of CIN cells; we suggest this is because CIN cells are particularly sensitive to entering mitosis with unrepaired DNA damage. If DNA damage or aneuploidy levels get too high, the JNK pathway is also used to drive apoptosis $[13,26,39]$. How the JNK pathway integrates multiple stress signals to decide between repair versus death is an area of active research $[40,41,42]$.

\subsection{Spindle assembly checkpoint mutations}

The best characterized cause of CIN is a failure in the spindle assembly checkpoint that allows segregation of chromosomes that are not correctly bioriented on the spindle (Figure 1C). There is abundant evidence that a defect in the spindle checkpoint in cell culture [43], animal models [44] or 
humans [45], leads to CIN. Analysis of mutations found in tumours suggests that complete loss of spindle checkpoint proteins is rare [46], presumably because that would generate an intolerably high level of instability. However there are numerous examples of tumours in which the checkpoint is aberrant, either in protein levels or localization $[44,47,48]$.

Using a defective spindle checkpoint as a CIN model has some advantages: the previously mentioned centrosomal and DNA damage models will tend to trigger checkpoints that promote cell cycle arrest and apoptosis rather than the desired unstable proliferation. In vertebrate systems, the level of spindle checkpoint disruption must be carefully controlled to avoid lethal levels of aneuploidy, but in Drosophila, the process of capturing the four chromosomes is sufficiently robust that even complete loss of the checkpoint can give viable animals [49]. This CIN model provides a perfect sensitized background for genetic screening in which even minor disruptions to genes needed for CIN cell survival can push these checkpoint compromised animals over the threshold of viability [11]. This screen tested the set of kinases and phosphatases and identified the JNK pathway and centrosomal signaling as key areas of CIN cell sensitivity. Although this type of viability screening has the benefit of being high-throughput, it has the drawback of a relatively non-specific phenotype: the death could be due to a range of developmental defects that would not be relevant to CIN tumours. However, further analysis allows confirmation that depleting the candidates by RNAi in proliferating CIN cells, such as the wing imaginal disc, can cause cell death [11]. This screen discarded mutations that were lethal in wild type as well as CIN animals, potentially missing some effective ways to kill CIN cells, however this approach should tend to identify more clinically useful approaches with fewer side effects on normal cells. Subsequent testing of good candidates in Drosophila tumour models [18] may allow confirmation that the genes in question are needed for the growth of bona fide CIN tumours. Our viability screen induced CIN by using RNAi to deplete the spindle checkpoint protein Mad2, but numerous alternative CIN models are available to confirm the generalizable effectiveness of candidates, including models depleting the checkpoint protein BubR1, or the cohesin $\operatorname{Rad} 21[38,50]$.

Another use of spindle checkpoint CIN models in Drosophila has been to explore the fate of CIN cells if apoptosis is prevented [12,13]. Although the spindle checkpoint is not strictly needed for Drosophila survival, checkpoint mutants lose many cells to apoptosis as they grow, so preventing apoptosis allows analysis of the behaviour of the most aneuploid and aberrant cells, which might also be found in apoptosis-resistant cancers. These were found to activate the JNK pathway and drop out of the epithelium, a metastatic behaviour seen in other similarly "undead" cells [39,51].

\subsection{Cytokinesis defects}

It has been noticed in human tumours that although they frequently show CIN and grossly aberrant karyotypes, in many cases the chromosome number seems to vary around a tetraploid rather than a diploid complement [52,53]. In some cases tetraploidy strongly predicts human tumour progression [54], and elegant studies in mice have shown that simply blocking one round of cytokinesis to give tetraploid cells can trigger tumorigenesis [55]. Drosophila is an excellent model system for studying cytokinesis - many of the genes and interactions that drive the process were discovered in flies [56-59]. Although there are numerous Drosophila RNAi lines that allow induction of cytokinesis failure and the generation of tetraploid cells, the relationship of tetraploidy to CIN development and tumorigenesis has not been intensively studied in flies [60]. Recent work showing 
the involvement of the Hippo pathway in tetraploid cell survival [61] may prompt further investigation in this area.

All these methods for generating CIN involve genetic disruption, but it is also possible to induce CIN chemically, for example by inhibiting kinesins with monastrol to cause monopolar spindle formation [62]. This approach has been used in vertebrate cell culture for some time, and has recently been developed in Drosophila by replacing the fly kinesin with the human version, which is sensitive to inhibition by small molecules [63]. Chemical induction of CIN has the advantage that it is convenient for developing high-throughput screening in culture, and by using Drosophila cells, it is convenient to then rapidly screen candidate hits in flies for in vivo phenotypes.

\section{Conclusion}

At the beginning of this review we posed a question of particular clinical interest: can we find targets that can be disrupted to specifically induce death in CIN cells without affecting normal dividing cells? Use of induced CIN models in Drosophila has allowed some significant progress to be made in this direction. It has been known for over a century that centrosomes are often aberrant in cancers [64], but studies in Drosophila have made it clear that just having the wrong number of centrosomes is not in itself enough to result in tumorigenesis [18], because either the spindle checkpoint can delay anaphase until a bipolar spindle has formed, or because the grossly aneuploid progeny will die by apoptosis. The striking exception to this generalization is also significant-in brains, neither the spindle checkpoint nor apoptosis are able to prevent tumorigenesis from neuroblasts that have lost their polarity cues. In these cells, too many or too few centrosomes or disruption of cortical polarity markers can all trigger failure to differentiate and hyperplasia that often progresses to metastatic tumours. It is not yet clear why neuroblasts are insensitive to genomic disruption that triggers apoptosis in other cells, but since human CIN tumours share this cell death resistance, either neuroblasts or disc cells with blocked apoptosis are appealing CIN models for looking at aneuploid cell behaviour [65].

The significance of centrosomes has been emphasized: in CIN cells even slight perturbation of the centrosomes tends to give cell death [11]. This is consistent with reports showing that the spindle checkpoint is needed to survive extra centrosomes [66]. The JNK pathway is also clearly implicated in CIN cell survival and proliferation. JNK is typically activated in response to cell stress, but it is important to recognize that it gives two possible outcomes: if the stress is low level it promotes repair, whereas if the stress is acute, JNK promotes apoptosis [40]. Consequently, blocking JNK signalling in CIN cells can either cause their death through failed DNA repair [11,38] or prevent their death in response to gross aneuploidy $[13,26]$. Although targeting either centrosomes or JNK signalling may allow effective manipulation of CIN cell fates, neither is ideal as a clinical target, due to their critical functions in normal cells. An alternative that may offer better clinical promise is to genetically or chemically target the metabolism of CIN cells. We have found that CIN cells are highly sensitive to RNAi knockdowns that give a range of mild metabolic perturbations that do not affect normal cells [50]. Some of these (e.g. Pas Kinase) may be amenable to chemical inhibition. It is known that aneuploid cells experience redox stress [67], so one possible hypothesis is that CIN cells, with their ongoing and varied aneuploidy, are close to the limits of their ability to buffer redox stress, and hence are vulnerable to metabolic intervention. Because tumours frequently display an aberrant metabolism as well as chromosomal instability, there are reasons to hope that metabolic therapy may be effective at 
generating tumour-specific apoptosis with minimal side effects [68].

There are a number of remaining challenges in understanding the causes and cellular responses to CIN. For example we still have no clear explanation for why the many varied genotypes generated in CIN populations so frequently seem to give the same stereotypical cell phenotype that includes overactive mitochondria, reactive oxygen species production, activation of the JNK pathway etc. It may be simply that any gene dosage variation gives protein folding stress [69], but we suspect that there may be other mechanisms that contribute to the response to aneuploidy. For example, many organisms can partially buffer gene dosage changes by down- or up-regulating the expression of genes in trisomic or monosomic DNA segments [70,71]. Several dosage compensation mechanisms exist for ensuring equal gene expression from sex chromosomes in males and females [72], and there are suggestions that extra somatic chromosomes can be shut down by nuclear compartmentalization [73], so it will be interesting to see whether CIN cells use similar processes to allow tolerance of gross aneuploidy. Because CIN develops fairly rapidly in Drosophila tumour models [25], they may be ideal for characterizing the acquisition of the changes that result in cells not only tolerating aberrant karyotypes, but also going on to proliferate and metastasize.

\section{Conflict of Interest}

All authors declare no conflicts of interest in this paper.

\section{References}

1. Lengauer C, Kinzler KW, Vogelstein B (1998) Genetic instabilities in human cancers. Nature 396: 643-649.

2. Weaver BA, Cleveland DW (2006) Does aneuploidy cause cancer? Curr Opin Cell Biol 18: 658-667.

3. Rao CV, Yamada HY (2013) Genomic instability and colon carcinogenesis: from the perspective of genes. Front Oncol 3: 130.

4. Duijf PHG, Benezra R (2013) The cancer biology of whole-chromosome instability. Oncogene 32: 4727-4736.

5. McGranahan N, Burrell RA, Endesfelder D, et al. (2012) Cancer chromosomal instability: therapeutic and diagnostic challenges. EMBO Rep 13: 528-538.

6. Carter SL, Eklund AC, Kohane IS, et al. (2006) A signature of chromosomal instability inferred from gene expression profiles predicts clinical outcome in multiple human cancers. Nat Genet 38: 1043-1048.

7. Bakhoum SF, Swanton C (2014) Chromosomal instability, aneuploidy, and cancer. Front Oncol 4: 161.

8. Roschke A, Kirsch I (2010) Targeting karyotypic complexity and chromosomal instability of cancer cells. Curr Drug Targets 11: 1341-1350.

9. Roschke AV, Lababidi S, Tonon G, et al. (2005) Karyotypic "state" as a potential determinant for anticancer drug discovery. Proc Natl Acad Sci U S A 102: 2964-2969.

10. Wallqvist A, Huang R, Covell DG, et al. (2005) Drugs aimed at targeting characteristic karyotypic phenotypes of cancer cells. Mol Cancer Ther 4: 1559-1568. 
11. Shaukat Z, Wong HWS, Nicolson S, et al. (2012) A Screen for Selective Killing of Cells with Chromosomal Instability Induced by a Spindle Checkpoint Defect. PLoS One 7: e47447.

12. Morais da Silva S, Moutinho-Santos T, Sunkel CE (2013) A tumor suppressor role of the Bub3 spindle checkpoint protein after apoptosis inhibition. J Cell Biol 201: 385-393.

13. Dekanty A, Barrio L, Muzzopappa M, et al. (2012) Aneuploidy-induced delaminating cells drive tumorigenesis in Drosophila epithelia. Proc Natl Acad Sci U S A 109: 20549-20554.

14. Silk AD, Zasadil LM, Holland AJ, et al. (2013) Chromosome missegregation rate predicts whether aneuploidy will promote or suppress tumors. Proc Natl Acad Sci U S A 110: E4134-E4141.

15. Foijer F, DiTommaso T, Donati G, et al. (2013) Spindle checkpoint deficiency is tolerated by murine epidermal cells but not hair follicle stem cells. Proc Natl Acad Sci US A 110: 2928-2933.

16. St Johnston D (2002) The art and design of genetic screens: Drosophila melanogaster. Nat Rev Genet 3: 176-188.

17. Brumby AM, Richardson HE (2005) Using Drosophila melanogaster to map human cancer pathways. Nat Rev Cancer 5: 626-639.

18. Gonzalez C (2013) Drosophila melanogaster: a model and a tool to investigate malignancy and identify new therapeutics. Nat Rev Cancer 13: 172-183.

19. Tipping M, Perrimon N (2014) Drosophila as a model for context-dependent tumorigenesis. $J$ Cell Physiol 229: 27-33.

20. Gladstone M, Su TT (2011) Chemical genetics and drug screening in Drosophila cancer models. $J$ Genet Genomics 38: 497-504.

21. Pastor-Pareja JC, Xu T (2013) Dissecting social cell biology and tumors using Drosophila genetics. Annu Rev Genet 47: 51-74.

22. Castellanos E, Dominguez P, Gonzalez C (2008) Centrosome dysfunction in Drosophila neural stem cells causes tumors that are not due to genome instability. Curr Biol 18: 1209-1214.

23. Basto R, Brunk K, Vinadogrova $T$, et al. (2008) Centrosome amplification can initiate tumorigenesis in flies. Cell 133: 1032-1042.

24. Kwon M, Godinho SA, Chandhok NS, et al. (2008) Mechanisms to suppress multipolar divisions in cancer cells with extra centrosomes. Genes Dev 22: 2189-2203.

25. Caussinus E, Gonzalez C (2005) Induction of tumor growth by altered stem-cell asymmetric division in Drosophila melanogaster. Nat Genet 37: 1125-1129.

26. Poulton JS, Cuningham JC, Peifer M (2014) Acentrosomal Drosophila Epithelial Cells Exhibit Abnormal Cell Division, Leading to Cell Death and Compensatory Proliferation. Dev Cell 30: 731-745.

27. Jüschke C, Dohnal I, Pichler P, et al. (2013) Transcriptome and proteome quantification of a tumor model provides novel insights into post-transcriptional gene regulation. Genome Biol 14: r133.

28. Purdy A, Su TT (2004) Telomeres: not all breaks are equal. Curr Biol 14: R613-R614.

29. Krüger A, Ralser M (2011) ATM Is a Redox Sensor Linking Genome Stability and Carbon Metabolism. Sci Signal 4: 4-6.

30. Li TS, Marbán E (2010) Physiological levels of reactive oxygen species are required to maintain genomic stability in stem cells. Stem Cells 28: 1178-1185. 
31. Pfau SJ, Amon A (2012) Chromosomal instability and aneuploidy in cancer: from yeast to man. EMBO Rep 13: 515-527.

32. Albrecht SC, Barata AG, Grosshans J, et al. (2011) In vivo mapping of hydrogen peroxide and oxidized glutathione reveals chemical and regional specificity of redox homeostasis. Cell Metab 14: 819-829.

33. Dronamraju R, Mason JM (2011) MU2 and HP1a regulate the recognition of double strand breaks in Drosophila melanogaster. PLoS One 6: e25439.

34. Chiolo I, Minoda A, Colmenares SU, et al. (2011) Double-strand breaks in heterochromatin move outside of a dynamic HP1a domain to complete recombinational repair. Cell 144: 732-744.

35. Burrell RA, McClelland SE, Endesfelder D, et al. (2013) Replication stress links structural and numerical cancer chromosomal instability. Nature 494: 492-496.

36. Bakhoum SF, Silkworth WT, Nardi IK, et al. (2014) The mitotic origin of chromosomal instability. Curr Biol 24: R148-R149.

37. Liu Y, Nielsen CF, Yao Q, et al. (2014) The origins and processing of ultra fine anaphase DNA bridges. Curr Opin Genet Dev 26C: 1-5.

38. Wong HW, Shaukat Z, Wang J, et al. (2014) JNK signaling is needed to tolerate chromosomal instability. Cell Cycle 13: 622-631.

39. McNamee LM, Brodsky MH (2009) p53-independent apoptosis limits DNA damage-induced aneuploidy. Genetics 182: 423-435.

40. Shaukat Z, Liu D, Hussain R, et al. (2014) The Role of JNK Signaling in Responses to Oxidative DNA damage. Curr Drug Targets [in press].

41. Karpac J, Biteau B, Jasper H (2013) Misregulation of an adaptive metabolic response contributes to the age-related disruption of lipid homeostasis in Drosophila. Cell Rep 4: $1250-1261$.

42. Christmann M, Kaina B (2013) Transcriptional regulation of human DNA repair genes following genotoxic stress: trigger mechanisms, inducible responses and genotoxic adaptation. Nucleic Acids Res 41: 8403-8420.

43. Thompson SL, Compton DA (2008) Examining the link between chromosomal instability and aneuploidy in human cells. $J$ Cell Biol 180: 665-672.

44. Perez de Castro I, de Carcer G, Malumbres M (2007) A census of mitotic cancer genes: new insights into tumor cell biology and cancer therapy. Carcinogenesis 28: 899-912.

45. Hanks S, Coleman K, Reid S, et al. (2004) Constitutional aneuploidy and cancer predisposition caused by biallelic mutations in BUB1B. Nat Genet 36: 1159-1161.

46. Wood LD, Parsons DW, Jones S, et al. (2007) The genomic landscapes of human breast and colorectal cancers. Science 318: 1108-1113.

47. Privette LM, Weier JF, Nguyen HN, et al. (2008) Loss of CHFR in human mammary epithelial cells causes genomic instability by disrupting the mitotic spindle assembly checkpoint. Neoplasia 10: 643-652.

48. Schvartzman JM, Sotillo R, Benezra R (2010) Mitotic chromosomal instability and cancer: mouse modelling of the human disease. Nat Rev Cancer 10: 102-115.

49. Buffin E, Emre D, Karess RE (2007) Flies without a spindle checkpoint. Nat Cell Biol 9: 565-572. 
50. Shaukat Z, Liu D, Choo A, et al. (2014) Chromosomal instability causes sensitivity to metabolic stress. Oncogene [Epub ahead of print].

51. Rudrapatna VA, Bangi E, Cagan RL (2013) Caspase signalling in the absence of apoptosis drives Jnk-dependent invasion. EMBO Rep 14: 172-177.

52. Olaharski AJ, Sotelo R, Solorza-Luna G, et al. (2006) Tetraploidy and chromosomal instability are early events during cervical carcinogenesis. Carcinogenesis 27: 337-343.

53. Margolis RL (2005) Tetraploidy and tumor development. Cancer Cell 8: 353-354.

54. Galipeau PC, Cowan DS, Sanchez CA, et al. (1996) 17p (p53) allelic losses, 4N (G2/tetraploid) populations, and progression to aneuploidy in Barrett's esophagus. Proc Natl Acad Sci U S A 93: 7081-7084.

55. Fujiwara T, Bandi M, Nitta M, et al. (2005) Cytokinesis failure generating tetraploids promotes tumorigenesis in p53-null cells. Nature 437: 1043-1047.

56. Ebrahimi S, Gregory SL (2011) Dissecting protein interactions during cytokinesis. Commun Integr Biol 4: 243-244.

57. D'Avino PP, Savoian MS, Glover DM (2005) Cleavage furrow formation and ingression during animal cytokinesis: a microtubule legacy. J Cell Sci 118: 1549-1558.

58. Somma MP, Ceprani F, Bucciarelli E, et al. (2008) Identification of Drosophila mitotic genes by combining co-expression analysis and RNA interference. PLoS Genet 4: e1000126.

59. Eggert US, Mitchison TJ, Field CM (2006) Animal cytokinesis: from parts list to mechanisms. Annu Rev Biochem 75: 543-566.

60. Fox DT, Duronio RJ (2013) Endoreplication and polyploidy: insights into development and disease. Development 140: 3-12.

61. Ganem NJ, Cornils H, Chiu SY, et al. (2014) Cytokinesis Failure Triggers Hippo Tumor Suppressor Pathway Activation. Cell 158: 833-848.

62. Lampson MA, Renduchitala K, Khodjakov A, et al. (2004) Correcting improper chromosome-spindle attachments during cell division. Nat Cell Biol 6: 232-237.

63. Salemi JD, McGilvray PT, Maresca TJ (2013) Development of a Drosophila cell-based error correction assay. Front Oncol 3: 187.

64. Holland AJ, Cleveland DW (2009) Boveri revisited: chromosomal instability, aneuploidy and tumorigenesis. Nat Rev Mol Cell Biol 10: 478-487.

65. Milán M, Clemente-Ruiz M, Dekanty A, et al. (2014) Aneuploidy and tumorigenesis in Drosophila. Semin Cell Dev Biol 28: 110-115.

66. Marthiens V, Piel M, Basto R (2012) Never tear us apart--the importance of centrosome clustering. J Cell Sci 125: 3281-3292.

67. Sheltzer JM, Torres EM, Dunham MJ, et al. (2012) Transcriptional consequences of aneuploidy. Proc Natl Acad Sci U S A 109: 12644-12649.

68. Gorrini C, Harris IS, Mak TW (2013) Modulation of oxidative stress as an anticancer strategy. Nat Rev Drug Discov 12: 931-947.

69. Oromendia AB, Dodgson SE, Amon A (2012) Aneuploidy causes proteotoxic stress in yeast. Genes Dev 26: 2696-2708.

70. Lundberg LE, Figueiredo MLA, Stenberg P, et al. (2012) Buffering and proteolysis are induced by segmental monosomy in Drosophila melanogaster. Nucleic Acids Res 40: 5926-5937.

71. Siegel JJ, Amon A (2012) New insights into the troubles of aneuploidy. Annu Rev Cell Dev Biol 28: 189-214. 
72. Ferrari F, Alekseyenko AA, Park PJ, et al. (2014) Transcriptional control of a whole chromosome: emerging models for dosage compensation. Nat Struct Mol Biol 21: 118-125.

73. Davidsson J, Veerla S, Johansson B (2013) Constitutional trisomy 8 mosaicism as a model for epigenetic studies of aneuploidy. Epigenetics Chromatin 6: 18.

(C) 2015, Stephen L. Gregory, et al., licensee AIMS Press. This is an open access article distributed under the terms of the Creative Commons Attribution License (http://creativecommons.org/licenses/by/4.0) 